\title{
Short duration of focal onset awareness and impaired awareness seizure are characteristic features of epilepsy in elderly patients
}

This article was published in the following Dove Press journal:

Neuropsychiatric Disease and Treatment

\author{
Ayataka Fujimoto' \\ Tohru Okanishi' \\ Yosuke Masuda' \\ Keishiro Sato' \\ Mitsuyo Nishimura' \\ Shimpei Baba' \\ Shinji Itamura' \\ Yoichiro Homma ${ }^{2}$ \\ Hideo Enoki' \\ 'Comprehensive Epilepsy Center. \\ Seirei Hamamatsu General Hospital, \\ Hamamatsu, Shizuoka, Japan; \\ ${ }^{2}$ Department of General Internal \\ Medicine, Seirei Hamamatsu General \\ Hospital, Hamamatsu, Shizuoka, Japan
}

Correspondence: Ayataka Fujimoto Comprehensive Epilepsy Center, Seirei Hamamatsu General Hospital, 2-12-12 Sumiyoshi, Nakaku, Hamamatsu, Shizuoka 430-8558, Japan

$\mathrm{Tel}+8 I 534742222$

Fax +8I 534757596

Email ataka_fuji@sis.seirei.or.jp
Purpose: Diagnosing epilepsy in the elderly population can be difficult due to mimicking symptoms. Furthermore, epileptic symptoms can also be masked by various symptoms. We hypothesized that elderly patients with epilepsy exhibit specific clinical features among the various symptoms.

Patients and methods: From 2009 to 2017, 177 patients who were older than 65 years were referred to our epilepsy center. Out of this group, the onset of symptoms occurred after reaching the age of 50 years in 152 of the patients, who were additionally being treated at our clinic. We divided their symptoms in accordance with their consciousness levels, which were defined as follows: full wakefulness level I, impaired awareness level II, and loss of consciousness level III. We also classified the duration of the symptoms as $<10$ seconds, $\geq 10$ seconds but $<1$ minute, $\geq 1$ minute but $<5$ minutes, $\geq 5$ minutes but $<10$ minutes, $\geq 10$ minutes but $<1$ hour, and $\geq 1$ hour.

Results: Among the 152 patients analyzed (mean age 72.9 years, standard deviation 6.71, range 65-92), 84 patients had epilepsy (epilepsy group) while 68 did not exhibit epilepsy (nonepilepsy group). For the consciousness levels, there were more level I patients in the nonepilepsy vs epilepsy group $(P<0.028)$, with symptom duration lasting $<1$ minute (sensitivity 0.857 , specificity 1 ) in the epilepsy group. In contrast, there were more level II patients in the epilepsy vs the nonepilepsy group $(P=0.015)$, with the duration of symptoms lasting $<1$ minute (sensitivity 0.8125 , specificity 0.930 ) in the epilepsy group. For the level III consciousness, convulsions were more commonly seen in the epilepsy group $(P=0.044)$.

Conclusion: Symptoms that last $<1$ minute with awareness and impaired awareness might be epileptic in the elderly population.

Keywords: epilepsy in elderly, $<1$-minute seizure, consciousness level, cognitive impairment, concomitant symptoms

\section{Introduction}

Epilepsy incidence exhibits bimodality in children and the elderly. ${ }^{1}$ It is known that incidence and prevalence of epilepsy increase with age. ${ }^{2-4}$ Along with the rapid population growth currently observed in the world, the elderly population is also rapidly increasing. ${ }^{5}$ However, at present, there has not been a large amount of research into epilepsy in the elderly. ${ }^{4}$ Moreover, it has been reported that the diagnosis of epilepsy in the elderly population can be difficult. ${ }^{2,6,7}$ The difficulties in diagnosing epilepsy in the elderly include the fact that epilepsy can be concomitant with post-stroke behavior, ${ }^{8}$ dementia, ${ }^{9}$ or the symptoms can mimic other psychiatric symptoms. ${ }^{10,11}$ Additionally, there are risks of syncope, hypo- and hyperglycemia, and transient ischemic attack in 
this patient population. Thus, overall these epileptic symptoms can be masked by various symptoms. The hypothesis of our present study was that epilepsy in elderly subjects exhibits specific clinical features that differ among the various other symptoms seen in this population. Furthermore, the main aim of this study was to review the consciousness level and duration of episodes during the clinical manifestation in elderly subjects.

\section{Patients and methods}

\section{Study design and ethical approval}

The Ethics Committee of Seirei Hamamatsu General Hospital in accordance with the principles of the Declaration of Helsinki approved the protocol for this study. The participants in this cross-sectional, observational, nonrandomized, intrasubjective comparison and case-controlled study were identified via a retrospective electronic chart review that was conducted from August 2009 to December 2017 at the Comprehensive Epilepsy Center, Seirei Hamamatsu General Hospital. Written informed consent was obtained from all patients.

\section{Patients}

From August 2009 to December 2017, 177 patients who were older than 65 years of age were referred to our Comprehensive Epilepsy Center. After reviewing the medical records, patients who fulfilled the following criteria were selected: 1) age older than 65 years, 2) onset of the symptoms occurred after reaching 50 years of age, and 3) have been treated at our clinic. Patients with onsets that occurred prior to reaching 50 years of age or that recurred after their 50's were excluded from the study.

\section{Medical questionnaire and medical interview}

\section{Primary outcome measures}

The primary outcome measures were the reviews of the consciousness level and duration of the symptoms.

All patients responded to a medical questionnaire form prior to undergoing a medical interview. Board-certified epilepsy specialists (AF and $\mathrm{KS}$ ) were responsible for obtaining the patient's detailed medical histories. Information on the medical history and symptoms were obtained not only from the actual patients but during telephone calls with eyewitnesses who directly observed the epilepsy episode. We also examined symptoms that could be observed in videos of the patient that were taken during the epilepsy episodes.

Patient symptoms were classified in accordance with their consciousness level. ${ }^{12,13}$ Consciousness level I was defined as symptoms that occurred while the patient was at a full wakefulness level. These included focal onset awareness seizure or simple partial seizure that occurred in epilepsy cases. At consciousness level I, it was required that the person was aware of self and environment during the seizure, even if immobile. The subjects were able to remember what had happened during the event. Consciousness level II was defined as symptoms that occurred while the patient was at an impaired awareness level. These included focal onset impaired awareness seizure or complex partial seizures that occurred during epilepsy cases. At consciousness level II, it was required that the person had an impaired awareness during any part of the event. These subjects exhibited impaired awareness of the events without realizing that they occurred. However, consciousness was maintained during this period. Consciousness level III was defined as symptoms that occurred while the patient had lost consciousness. These included bilateral tonic-clonic seizure or secondary generalized seizure that occurred during epilepsy cases. At consciousness level III, it was required that the patients had completely lost consciousness and were not awakened by external stimuli. During the level III evaluations, we also reviewed whether or not the patients exhibited convulsions.

In addition to determining the different consciousness levels, we also reviewed the duration of symptoms. The duration of the symptoms was divided as follows:

1. $<10$ seconds

2. $\geq 10$ seconds but $<1$ minute

3. $\geq 1$ minute but $<5$ minutes

4. $\geq 5$ minutes but $<10$ minutes

5. $\geq 10$ minutes but $<1$ hour

6 . $\geq 1$ hour.

\section{Secondary outcome measurements}

Secondary outcome measurements investigated included the cognitive impairment, psychiatric symptoms, whether or not the patient was able to drive, traffic accidents caused by the symptoms, diagnosis, etiology of the symptoms, treatment, and amnesia. To survey the cognitive impairment and psychiatric symptoms, we obtained referral letters from each specialist who saw the patient. For the driving and traffic accident surveys, we interviewed the patients, their family, and previous physicians in addition to checking information from referral letters.

\section{Diagnosis and treatment Diagnosis}

All patients underwent brain 3.0-T magnetic resonance image and electroencephalography (EEG) evaluations. 
The EEGs were objectively read by board-certified clinical neurophysiologists in EEG (AF, KS, TO, and EH). After reading the EEGs, the neurophysiologists in EEG reviewed all of the findings and submitted reports on each of the subjects. If further examinations were required, patients underwent long-term video EEG (VEEG), single photon emission tomography, 2-[ $\left.{ }^{18} \mathrm{~F}\right]$ fluoro-2-deoxy-D-glucose positron emission tomography, and neuropsychological evaluation. Epilepsy was diagnosed by board-certified epileptologists (AF and KS) on the basis of the patient's clinical information, which included medical history, EEG reports, 3.0-T magnetic resonance imaging, VEEG, single photon emission tomography, and positron emission tomography. When we encountered difficulties in diagnosing the epilepsy, patients were further discussed during Comprehensive Epilepsy Center conferences. At these conferences, patients were discussed by the authors and other epileptologists (TO, YM, MN, SB, SI, and HE).

\section{Treatment}

After the diagnosis of epilepsy, we appropriately adjusted the patient's antiepilepsy drugs (AEDs), performed epilepsy surgery, vagus nerve stimulation therapy, and, where necessary, took a wait-and-see (WAS) approach considering their social background. If patients were diagnosed as being nonepileptic, we took a WAS approach or sent them to each of the study specialists that included cardiologists, endocrinologists, psychiatrists, etc, for further investigation, treatment, and follow-up.

\section{Statistical analysis}

We statistically compared the epilepsy and nonepilepsy groups by using the Mann-Whitney rank-sum test, chisquared test, and Fisher's exact test, as appropriate. Statistical significance was set at $P<0.05$. The cutoff value was calculated by the receiver operating characteristic (ROC) curve analysis. All analyses were performed using Sigma Plot 14 software (Systat Software, San Jose, CA, USA).

\section{Results}

\section{Clinical information}

Among the 177 patients who were referred to our epilepsy center, 152 patients met the inclusion criteria (mean age 72.9 years, standard deviation 6.71, range 65-92). Among the enrolled patients, 84 ( 34 females and 50 males) were diagnosed with epilepsy (epilepsy group) while 68 (34 females and 34 males) were diagnosed as not having epilepsy (nonepilepsy group). There was no statistical difference between the groups in terms of age $(P=0.621)$ and sex $(P=0.493)$. Table 1 presents the clinical information for the patients.

\section{Primary outcome measures Consciousness level I}

There were 6 patients who exhibited clinical manifestations under wakefulness ( 3 motor, 2 sensory, and 1 aphasia with motor manifestation) in the epilepsy group while there were 14 patients in the nonepilepsy group. There were more patients in the nonepilepsy vs the epilepsy group $(P<0.028)$.

The duration in the epilepsy group was shorter than that observed in the nonepilepsy group $(P<0.001)$. ROC curve analysis indicated that the cutoff value was level III. This meant that the duration of the epileptic seizure in elderly patients was $<1$ minute (sensitivity $=1-$ false-positive fraction $[\mathrm{FPF}]=1-0.429=0.857$, specificity $=$ true positive fraction $[\mathrm{TPF}]=1.00)$.

One patient with epilepsy (Patient No. 76) exhibited aphasia and hand twitching under wakefulness followed by bilateral tonic-clonic convulsion. This patient was classified as level III.

\section{Consciousness level II}

There were 57 patients who exhibited a loss of awareness in the epilepsy group, while there were 32 patients who exhibited attention, concentration and performance impairment, etc, in the nonepilepsy group. The difference between these groups was statistically significant $(P=0.015)$. In addition, impaired awareness was more often seen in the epilepsy vs the nonepilepsy group.

There was a shorter duration in the epilepsy vs the nonepilepsy group $(P<0.001)$. ROC curve analysis showed that the cutoff value was level III. This indicated that the duration of the epileptic seizure in the elderly patients was $<1$ minute (sensitivity $=1-\mathrm{FPF}=1-0.1875=0.8125$, specificity $=$ TPF $=0.930$ ).

There were seven patients who exhibited focal onset impaired awareness seizure followed by bilateral tonic-clonic seizure. These seven patients were classified as level III.

\section{Consciousness level III}

There were 22 patients in the epilepsy and 28 patients in the nonepilepsy group who lost consciousness. However, there was no statistically significant difference between these two groups $(P=0.075)$. There was also no statistical difference for the duration between the two groups $(P=0.748)$. In terms of convulsion, there were 22 patients in the epilepsy and 8 in the nonepilepsy group who exhibited generalized convulsions. 


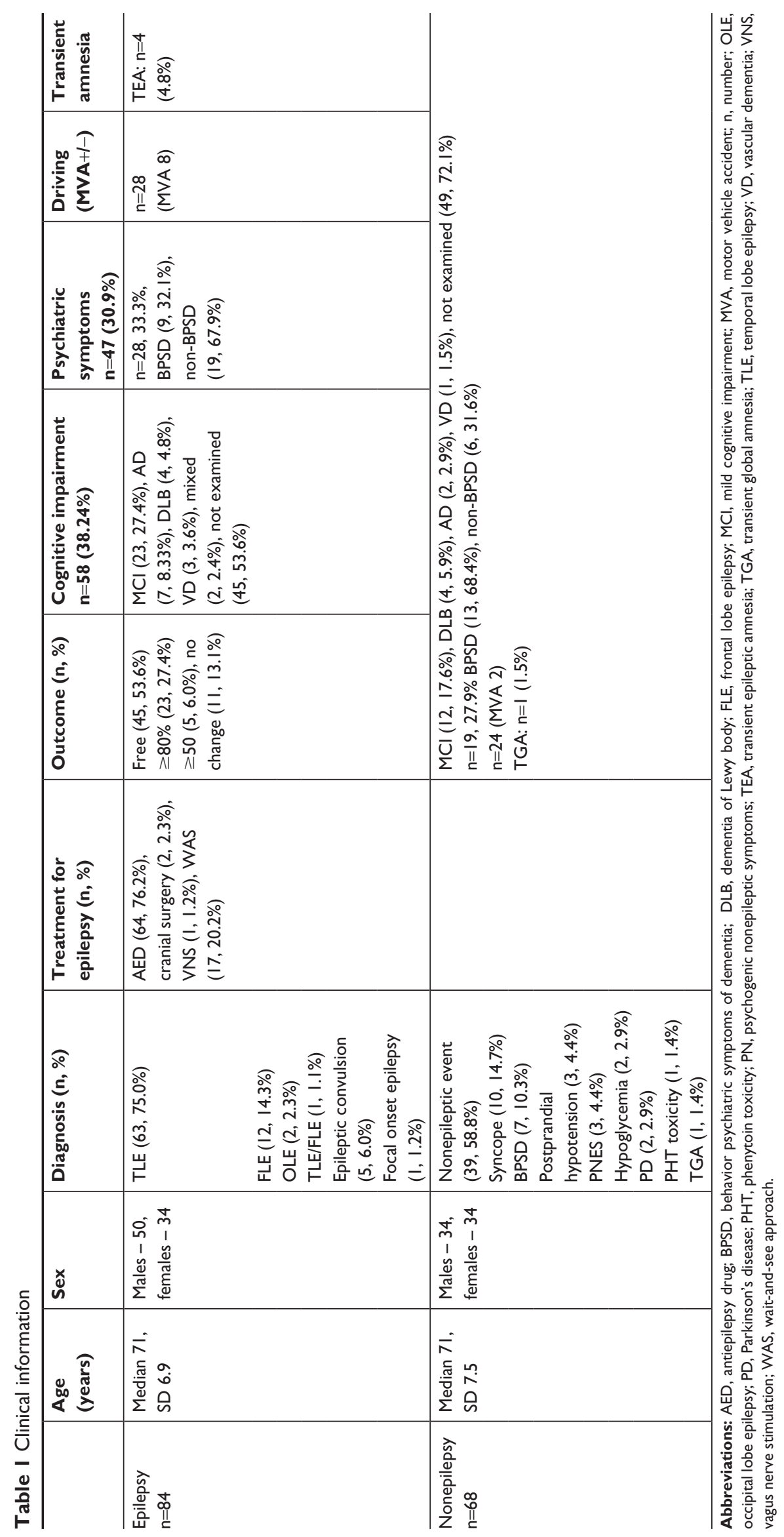


group. The difference between the groups was not statistically significant.

There were $23(59 \%)$ patients with mild cognitive impairment (MCI), 7 (17.9\%) with Alzheimer disease (AD), 4 (10.3\%) with dementia with Lewy body (DLB), 3 (7.7\%) with vascular dementia, and $2(5.1 \%)$ with mixed dementia in the epilepsy group.

There were 12 (63.2\%) MCI, 4 (21.1\%) DLB, 2 (10.5\%) $\mathrm{AD}$, and $1(4.2 \%)$ vascular dementia in the nonepilepsy group.

There was no statistically significant difference between the two groups $(P=0.916)$. There was also no statistically significant difference found for the different forms of dementia (MCI $P=0.321$, AD $P=0.703$, DLB $P=0.246$, vs $P=1.000$ ).

\section{Psychiatric symptoms}

There were 28 (33.3\%) patients in the epilepsy and 19 (27.9\%) in the nonepilepsy group who exhibited psychiatric symptoms. The difference between the groups was not statistically significant $(P=0.590)$. There were $9(32.1 \%)$ behavioral and psychological symptoms of dementia (BPSD) in the epilepsy and $13(68.4 \%)$ in the nonepilepsy group. The difference between the groups was statistically significant $(P=0.019)$.

\section{Driving}

There were 28 patients who continued to drive at the time of diagnosis in the epilepsy and 24 in the nonepilepsy group, which was not statistically significant $(P=0.725)$. There were eight patients with traffic accidents that were associated with their symptoms in the epilepsy and two in the nonepilepsy group, which was not statistically significant $(P=0.086)$.

\section{Diagnosis}

In the Epilepsy group, there were $63(75.0 \%)$ patients with temporal lobe epilepsy (TLE), 12 (14.3\%) with frontal lobe epilepsy (FLE), 2 (2.3\%) with occipital lobe epilepsy, 1 (1.1\%) with temporofrontal lobe epilepsy, $5(6.0 \%)$ with epileptic convulsion, and $1(1.1 \%)$ with focal onset epilepsy. There was no significance difference between the TLE and FLE for the duration $(P=0.890)$ and consciousness level $(P=0.304)$. In the nonepilepsy group, there were 39 (58.8\%) nonepileptic patients with an unknown etiology, 10 (14.7\%) with syncope, 7 (10.3\%) with BPSD, 3 (4.4\%) with postprandial hypotension, $3(4.4 \%)$ with psychogenic nonepileptic spells, 2 (2.9\%) with hypoglycemia, 2 (2.9\%) with Parkinson's disease or Parkinson's syndrome, 1 (1.4\%) with phenytoin toxicity, and $1(1.4 \%)$ with transient global

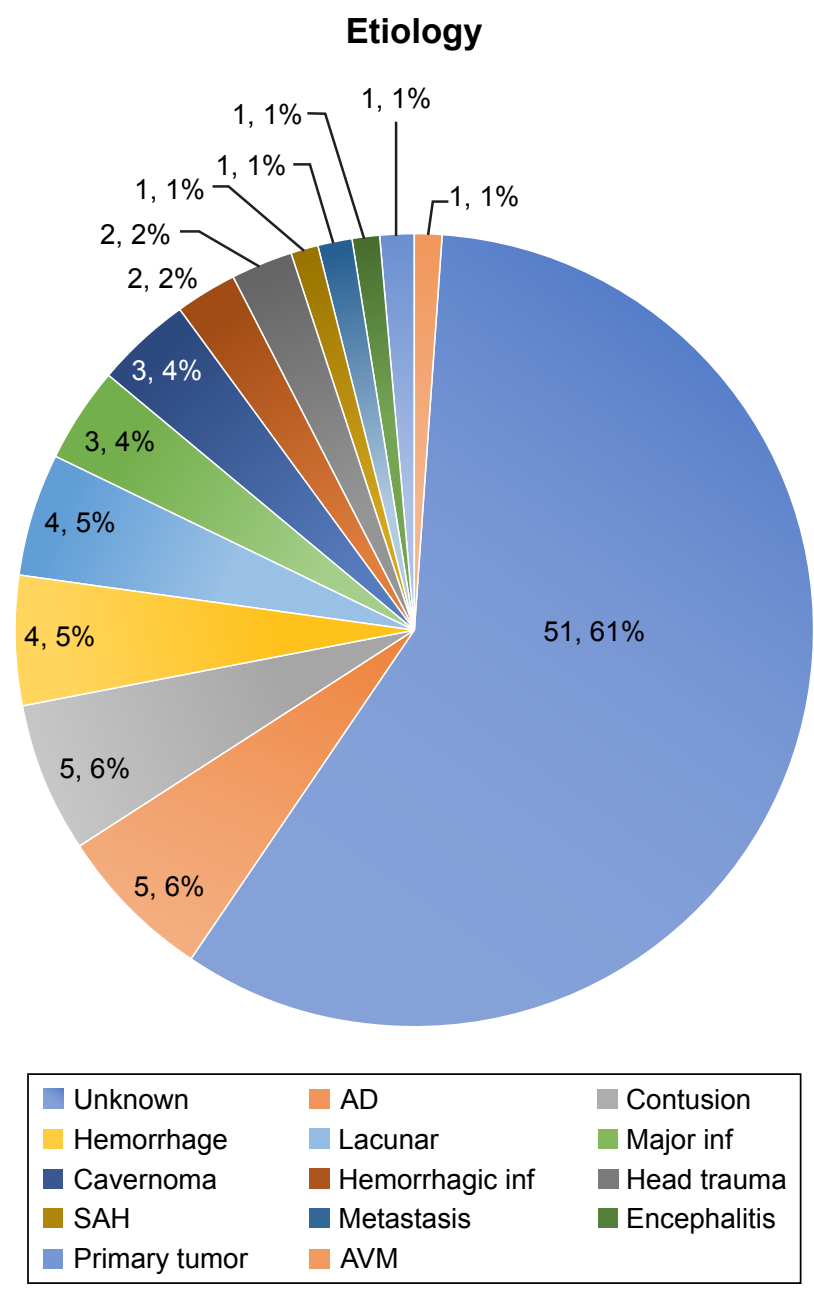

Figure 2 Etiology of epilepsy.

Notes: This figure presents the etiology of epilepsy. A total of $61 \%$ of these patients exhibited epilepsy of an unknown etiology followed by $A D$. Lacunar infarction (lacunar), major vessel infarction (major inf), hemorrhagic infarction (hemorrhagic inf), SAH, AVM.

Abbreviations: AD, Alzheimer's disease; AVM, arteriovenous malformation; $\mathrm{SAH}$, subarachanoid hemorrhage.

amnesia. Figure 2 shows the breakdown of the etiology in the nonepilepsy group.

\section{Treatment}

AED adjustment was performed in 64 (76.2\%) patients. Open cranial surgery was performed in $2(2.3 \%)$ patients, while $1(1.2 \%)$ underwent vagus nerve stimulation therapy and $17(13.1 \%)$ were placed in the WAS approach category.

There were 45 (53.6\%) patients who achieved seizure freedom, with $23(27.4 \%)$ classified as more than $80 \%$ responders and $5(6.0 \%)$ who were more than $50 \%$ responders. No appreciable change was observed in $11(13.1 \%)$ patients.

\section{Amnesia}

The diagnosis of transient epileptic amnesia (TEA) was made on the basis of the following criteria: 1) there was a history of 
recurrent witnessed episodes of transient amnesia; 2) cognitive functions other than memory were judged to be intact during typical episodes by a reliable witness; and 3) there was evidence for a diagnosis of epilepsy. ${ }^{14}$ There were four patients who met the criteria for inclusion in the epilepsy group, and one patient who was diagnosed as transient global amnesia in the nonepilepsy group.

\section{Discussion \\ Primary outcome measures}

The cutoff value for the duration in elderly with epilepsy was $<1$ minute when our results classified these patients as level I and II consciousness. Although, the duration of focal onset impaired awareness seizures have been reported to typically last more than 1 minute, ${ }^{15,16}$ symptoms that last $<1$ minute during impaired awareness might actually be epileptic in the elderly population.

However, it is our belief that it could be fairly difficult for elderly to precisely describe their aura. Thus, this could be the reason why only six patients with wakeful consciousness level (level I) were diagnosed as having epilepsy. Based on these findings, this suggests that diagnosing focal onset awareness seizure in the elderly population could be challenging. Furthermore, since the duration of awareness seizures generally vary, ${ }^{16,17}$ and the short duration in wakefulness seen in our study might actually be a feature of elderly epilepsy. However, in order to avoid any misdiagnosis, the presence of long-lasting nonconvulsive status epilepticus also should be taken into consideration when evaluating these types of patients. ${ }^{18}$

As consciousness level and duration of the symptoms could not be used to determine the difference between epileptic and nonepileptic symptoms for the consciousness level III, it might be possible to use the presence of convulsions as a factor to distinguish between the symptoms.

The hypothesis of our present study was that there are specific clinical features associated with elderly subjects with epilepsy. Our results did show that awareness and impaired awareness seizures with less than a 1-minute duration appear to be a specific clinical feature that is associated with elderly patients with epilepsy.

One of the limitations of this study was that the durations of the symptoms were not always meticulously determined during the observations in this study. In fact, while many of the symptoms were recorded by the videos taken of the patient, others came from eyewitness accounts of the symptoms, which at times could be very subjective in nature. To obtain more accurate information on the duration, a further study that performs VEEG and accurately measures the duration in seconds will need to be undertaken in the future.

\section{Secondary outcome measures}

\section{Concomitant symptoms}

Cognitive impairment was not significantly different in each group. However, BPSD was more commonly seen in the nonepilepsy group. This indicates that there were more MCI with BPSD in the nonepilepsy group. ${ }^{19}$ Additionally, it is known that there is a higher prevalence of psychosis found in patients with epilepsy compared with the general population. ${ }^{20}$ This could potentially be explained by the fact that there were relatively many more non-BPSD patients in the epilepsy group vs the number of BPSD patients in the nonepilepsy group. As cognitive impairment and psychiatric symptoms were evenly seen in both groups, this makes the diagnosis of epilepsy difficult. In addition, it is our belief that MCI with BPSD also makes it much more difficult to determine this diagnosis in the elderly population.

\section{Diagnosis and treatment}

TLE (75\%) followed by FLE were the most commonly seen forms of epilepsy in this study. Since TLE is the most common epilepsy observed in adults, it is likely that the elderly population will also exhibit the same level of occurrence. ${ }^{3}$ Amyloid deposition is one of the common conditions seen in both $\mathrm{AD}$ and cerebral hemorrhage. ${ }^{21}$ Based on these findings, it has been presumed that the peripheral symptoms related to these disease states might also complicate making a diagnosis for these types of diseases. ${ }^{22}$

Although the success of treatments aimed at achieving seizure freedom in patients are as low as $53.6 \%$, this level was considered to be refractory enough for these treatments to be introduced for use in our epilepsy center. Panayiotopoulos ${ }^{23}$ has reported that elderly patients are more likely to suffer from drug-induced disease than derive any benefits from ill-advised AED prescribing. Therefore, when treating elderly patients with epilepsy, it is important to not merely achieve complete seizure freedom, but also consider the balance between treatment efficacy and the adverse effect of AEDs.

\section{Amnesia}

A previous study reported on a patient with TEA who did not exhibit any epileptic seizures. ${ }^{24}$ However, all four patients with TEA in our study had impaired awareness seizure. Thus, these types of cases might be missed impaired awareness seizure due to the very short duration of the seizure. 


\section{Driving and traffic accidents}

There was no significance difference observed between the epilepsy and nonepilepsy groups in terms of driving $(P=0.725)$. This is likely due to the fact that there are overall issues with driving in the elderly population. Moreover, we also found that there was no statistically significant difference in both groups for traffic accidents related to their symptoms $(P=0.086)$. Thus, these results appear to suggest that epilepsy cannot be definitively cited as a cause of traffic accidents involving elderly.

\section{Conclusion}

Symptoms that last $<1$ minute with awareness and impaired awareness might be epileptic in the elderly population.

\section{Ethical publication statement}

We confirm that we have read the Journal's position on issues involved in ethical publication and affirm that this report is consistent with those guidelines.

\section{Author contributions}

All authors made substantial contributions to conception and design, acquisition of data, or analysis and interpretation of data; took part in drafting the article or revising it critically for important intellectual content; gave final approval of the version to be published; and agree to be accountable for all aspects of the work.

\section{Disclosure}

The authors report no conflicts of interest concerning the patients or methods used in this study or the findings specified in this paper. All authors certify that they have no affiliations with or involvement in any organization or entity with any financial interest (such as honoraria; educational grants; participation in speakers' bureaus; membership, employment, consultancies, stock ownership, or other equity interest; and expert testimony or patent-licensing arrangements), or nonfinancial interest (such as personal or professional relationships, affiliations, knowledge, or beliefs) in the subject matter or materials discussed in this manuscript.

\section{References}

1. Hauser WA, Annegers JF, Kurland LT. Incidence of epilepsy and unprovoked seizures in Rochester, Minnesota: 1935-1984. Epilepsia. 1993; 34(3):453-458.

2. Brodie MJ, Elder AT, Kwan P. Epilepsy in later life. Lancet Neurol. 2009;8(11):1019-1030.

3. Tanaka A, Akamatsu N, Shouzaki T, et al. Clinical characteristics and treatment responses in new-onset epilepsy in the elderly. Seizure. 2013;22(9):772-775.
4. Hernández-Ronquillo L, Adams S, Ballendine S, Téllez-Zenteno JF. Epilepsy in an elderly population: Classification, etiology and drug resistance. Epilepsy Res. 2018;140:90-94.

5. Guilbert JJ. The World Health report 2006: Working together for health. Educ Health. 2006;19(3):385-387.

6. Sirven JI, Ozuna J. Epilepsy Foundation of America. Diagnosing epilepsy in older adults: what does it mean for the primary care physician? Geriatrics. 2005;60(10):30-35.

7. Austin J, Abdulla A. Identifying and managing epilepsy in older adults. Nurs Times. 2013;109(3):20-23.

8. Tanaka T, Yamagami H, Ihara M, et al. Seizure Outcomes and Predictors of Recurrent Post-Stroke Seizure: A Retrospective Observational Cohort Study. PLoS One. 2015;10(8):e0136200.

9. Vossel KA, Tartaglia MC, Nygaard HB, Zeman AZ, Miller BL. Epileptic activity in Alzheimer's disease: causes and clinical relevance. Lancet Neurol. 2017;16(4):311-322.

10. Naeije G, Bachir I, Gaspard N, Legros B, Pepersack T. Epileptic activities are common in older people with delirium. Geriatr Gerontol Int. 2014;14(2):447-451.

11. Naeije G, Depondt C, Meeus C, Korpak K, Pepersack T, Legros B. EEG patterns compatible with nonconvulsive status epilepticus are common in elderly patients with delirium: a prospective study with continuous EEG monitoring. Epilepsy Behav. 2014;36:18-21.

12. Di Perri C, Stender J, Laureys S, Gosseries O. Functional neuroanatomy of disorders of consciousness. Epilepsy Behav. 2014;30:28-32.

13. Fisher RS, Cross JH, French JA, et al. Operational classification of seizure types by the International League Against Epilepsy: Position Paper of the ILAE Commission for Classification and Terminology. Epilepsia. 2017;58(4):522-530.

14. Zeman AZ, Boniface SJ, Hodges JR. Transient epileptic amnesia: a description of the clinical and neuropsychological features in 10 cases and a review of the literature. J Neurol Neurosurg Psychiatry. 1998; 64(4):435-443.

15. Panayiotopoulos CP. The Epilepsies: Seizures, Syndromes and Management. Oxfordshire, UK: Bladon Medical Publishing; 2005.

16. Villanueva V, Serratosa JM. Temporal lobe epilepsy: clinical semiology and age at onset. Epileptic Disord. 2005;7(2):83-90.

17. Walker MC, Smith SJ, Sisodiya SM, Shorvon SD. Case of simple partial status epilepticus in occipital lobe epilepsy misdiagnosed as migraine: clinical, electrophysiological, and magnetic resonance imaging characteristics. Epilepsia. 1995;36(12):1233-1236.

18. Meierkord H, Holtkamp M. Non-convulsive status epilepticus in adults: clinical forms and treatment. Lancet Neurol. 2007;6(4):329-339.

19. Lyketsos CG, Lopez O, Jones B, Fitzpatrick AL, Breitner J, Dekosky S. Prevalence of neuropsychiatric symptoms in dementia and mild cognitive impairment: results from the cardiovascular health study. JAMA. 2002;288(12):1475-1483.

20. Clancy MJ, Clarke MC, Connor DJ, Cannon M, Cotter DR. The prevalence of psychosis in epilepsy; a systematic review and meta-analysis. BMC Psychiatry. 2014;14:75.

21. Keable A, Fenna K, Yuen HM, et al. Deposition of amyloid beta in the walls of human leptomeningeal arteries in relation to perivascular drainage pathways in cerebral amyloid angiopathy. Biochim Biophys Acta. 1862;2016(5):1037-1046.

22. Ramsay RE, Macias FM, Rowan AJ. Diagnosing epilepsy in the elderly. Int Rev Neurobiol. 2007;81:129-151.

23. Panayiotopoulos CP. Principles of therapy in the epilepsies. A Clinical Guide to Epileptic Syndromes and Their Treatment. Berlin: Springer; 2010:173-235.

24. Ukai K, Watanabe M. Transient epileptic amnesia without epileptic seizures: proposal of a new entity. Psychogeriatrics. 2017;17(6): $491-492$. 
Neuropsychiatric Disease and Treatment

Dovepress

\section{Publish your work in this journal}

Neuropsychiatric Disease and Treatment is an international, peerreviewed journal of clinical therapeutics and pharmacology focusing on concise rapid reporting of clinical or pre-clinical studies on a range of neuropsychiatric and neurological disorders. This journal is indexed on PubMed Central, the 'PsycINFO' database and CAS,

and is the official journal of The International Neuropsychiatric Association (INA). The manuscript management system is completely online and includes a very quick and fair peer-review system, which is all easy to use. Visit http://www.dovepress.com/testimonials.php to read real quotes from published authors.

Submit your manuscript here: http://www.dovepress.com/neuropsychiatric-disease-and-treatment-journal 